\title{
Onset of chaotic advection in open flows
}

\author{
J. J. Benjamin Biemond, ${ }^{1}$ Alessandro P. S. de Moura, ${ }^{2}$ György Károlyi, ${ }^{2}$ Celso Grebogi, ${ }^{2}$ and Henk Nijmeijer ${ }^{1}$ \\ ${ }^{1}$ Department of Mechanical Engineering, Eindhoven University of Technology, P.O. Box 513, 5600 MB Eindhoven, The Netherlands \\ ${ }^{2}$ College of Physical Sciences, King's College, University of Aberdeen, Aberdeen AB24 3UE, United Kingdom
}

(Received 21 February 2008; revised manuscript received 2 June 2008; published 25 July 2008)

\begin{abstract}
In this paper we investigate the transition to chaos in the motion of particles advected by open flows with obstacles. By means of a topological argument, we show that the separation points on the surface of the obstacle imply the existence of a saddle point downstream from the obstacle, with an associated heteroclinic orbit. We argue that as soon as the flow becomes time periodic, these orbits give rise to heteroclinic tangles, causing passively advected particles to experience transient chaos. The transition to chaos thus coincides with the onset of time dependence in open flows with stagnant points, in contrast with flows with no stagnant points. We also show that the nonhyperbolic nature of the dynamics near the walls causes anomalous scalings in the vicinity of the transition. These results are confirmed by numerical simulations of the two-dimensional flow around a cylinder.
\end{abstract}

DOI: 10.1103/PhysRevE.78.016317

PACS number(s): 47.52. $+\mathrm{j}, 05.45 .-\mathrm{a}, 47.10 . \mathrm{Fg}$

Two-dimensional flow around an obstacle is stationary for low Reynolds numbers, and the dynamics of passively advected particles is integrable. For higher Reynolds numbers, the flow displays a time-periodic shedding of vortices, leading to the formation of the von Kármán vortex street [1]. In this nonstationary regime, particles commonly experience transient chaotic motion [2,3]. Chaotic advection is present in many important systems, especially in environmental flows such as the atmosphere and the oceans [4]. It is relevant for key environmental phenomena such as the depletion of the ozone layer [5] and plankton blooms [6-9].

A natural question to ask is whether the transition to chaos in open flows coincides with the transition from stationary to time-dependent flow; does chaotic advection appear as soon as the flow becomes time dependent? In this paper, the transition from the regular to the chaotic regime is investigated with a simplified analytical flow model, which incorporates the main features of the flow dynamics near the transition from stationarity to time dependence. The main goal is to determine if and under what conditions the transition from regular to chaotic advection is simultaneous with the transition from stationary to time-dependent fluid motion, and to identify the dynamical mechanisms governing this transition. Our main result is that the nature of the transition to chaotic advection, and when it takes place, depend on whether or not there are stagnant points in the flow-points where the flow velocity is zero, such as on the surface of walls and obstacles. In the absence of stagnant points, the advection dynamics remains regular immediately after the transition to nonstationarity. This means that there is a range of Reynolds numbers, above the critical value for the onset of nonstationarity, for which the advection remains regular, even though the flow is time dependent. In this case, chaotic advection only sets in when the strength of time-dependent perturbation of the velocity field exceeds a certain threshold. If, on the other hand, stagnant points are present in the flow, we show that the motion of particles becomes chaotic as soon as the time dependence sets in. We argue in this work that this qualitative difference between the two cases is due to the presence of the stagnant points, resulting in the existence of heteroclinic orbits. These orbits break up and produce a het- eroclinic tangle even for arbitrarily small time-dependent perturbations of the stationary flow, leading to chaos. This situation is very common in realistic flows, since stagnant points always exist in the presence of walls.

We also study in this work the effects of the nonhyperbolic nature of the advection on the dynamics, a result from the presence of walls in the flow. Due to the no-slip boundary conditions imposed on the Navier-Stokes equations, the surface of an obstacle consists of a set of fixed points with zero eigenvalues. Therefore the advection dynamics is nonhyperbolic when obstacles or other boundaries are present. The heteroclinic orbits which exist before the time dependence sets in break into heteroclinic tangles. Once this breakup occurs, particles coming from the outside can access the chaotic region present on the wake of the obstacle. In hyperbolic systems, the number of particles $N_{v}$ entering the region excluded by the previously existing separatrix is expected to depend on a bifurcation parameter $p$ (such as the Reynolds number) as $N_{v} \sim\left(p-p_{c}\right)^{\gamma}$, with $p_{c}$ being the critical parameter value (it can be thought of as the Reynolds number for which the flow becomes time dependent). Here, $\gamma$ is a critical exponent, which depends on the eigenvalues of the fixed points $[10,11]$. We find that due to the presence of the surface consisting of fixed points and the nonhyperbolic dynamics close to this surface, this relationship does not hold in our case. We have instead an anomalous law of the form $N_{v}$ $\sim \exp \left[k\left(p-p_{c}\right)^{-\eta}\right]$, where $k$ and $\eta$ are constants.

In order to analyze the transition from a stationary to a time-dependent flow, we use an analytic model for a twodimensional incompressible flow with a cylindrical obstacle, adapted from a model introduced in [2]. This model can serve as a prototype for the flow of other bluff-body obstacles in a uniform background flow. For two-dimensional (2D) incompressible flows, the velocity $(\dot{x}, \dot{y})$ of an advected particle is given by a time-dependent stream function $\psi(x, y, t)$, such that

$$
\dot{x}(t)=\frac{\partial}{\partial y} \psi(x, y, t), \quad \dot{y}(t)=-\frac{\partial}{\partial x} \psi(x, y, t) .
$$

We use for $\psi$ the form introduced in Ref. [2], which is an analytical approximation to the time-dependent, periodic re- 


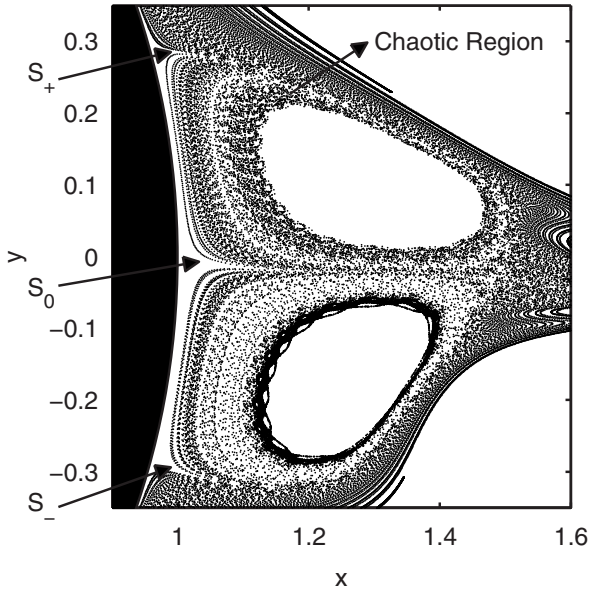

FIG. 1. Part of a numerically calculated streakline for $w=2$ in the wake of the obstacle. Ten thousand particles were injected per period at position $(x, y)=(-3,0)$ into the flow, and their subsequent positions were plotted every period, at times given by $t \bmod T=0$, until they leave the time-dependent region and travel downstream. The solid black area on the left is the cylindrical obstacle.

gime of the flow, with two vortices being shed from the back of the cylinder in an alternate manner:

$$
\psi(x, y, t)=f(x, y) g(x, y, t) .
$$

Here $f(x, y)$ accounts for the no-slip boundary conditions on the surface of the cylinder of unit radius:

$$
f(x, y)=1-\exp \left[-a\left(\sqrt{x^{2}+y^{2}}-1\right)^{2}\right],
$$

with $a$ determining the width of the boundary layer. The factor $g(x, y, t)$ describes the two vortices and the background flow:

$$
g(x, y, t)=-w h_{1}(t) g_{1}(x, y, t)+w h_{2}(t) g_{2}(x, y, t)+u_{0} y s(x, y),
$$

where $h_{i}(t), i=1,2$ describe the growth and decay of the vortex amplitudes that are periodic with a period of $T=1$ and vortex strength $w$, and $g_{i}(x, y, t)$ determine the spatial structure of the vortices which are moving in the positive $x$ direction, as given in Ref. [2]. The term $s(x, y)$ corresponds to the shielding effect of the cylinder on the uniform background flow of velocity $u_{0}$. The parameters of this flow model are set in [2] to fit the simulation results of the Navier-Stokes equations for a constant background velocity in a channel. We use the same parameter values except for the vortex strength $w$, which we choose as our bifurcation parameter. For $w=0$, the velocity field is stationary; as $w$ increases from 0 , the flow becomes time dependent. This flow model emulates the qualitative behavior of the real system. We note, however, that in the limit of $w \rightarrow 0$, the model represents two moving vortices with infinitesimally small vorticity, whereas in real flows, two stationary vortices of finite strength exist before the transition to time-dependent fluid motion. However, by using this model, we uncover general features of the transition to chaotic advection in time-dependent Hamiltonian flows. To confirm that our results are not dependent on the details of the model, we also consider later an alternative
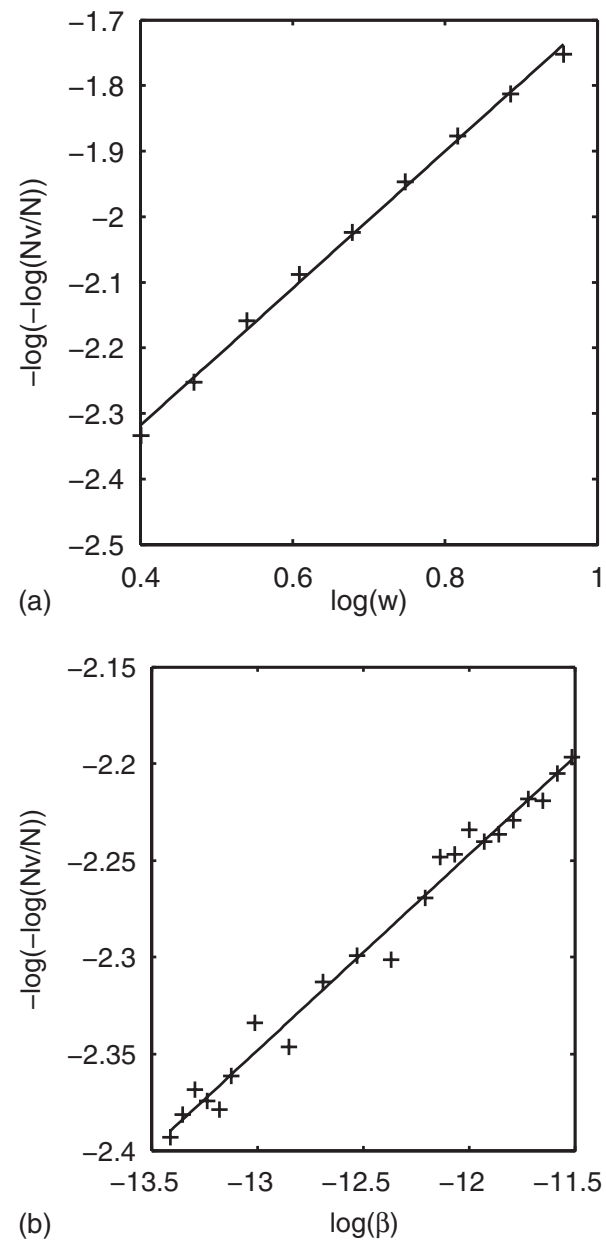

FIG. 2. Ratio of the number $N_{v}$ of particles entering the wake to all $N$ particles injected into the flow at time $t=0$, as a function of the bifurcation parameter, with random initial positions distributed uniformly in the region $-16.0<x<-1.1,-0.05<y<0.05$. The function $\log ($.) denotes the natural logarithm. In (a) the original model with bifurcation parameter $w$ is investigated by injecting $N=1$ $\times 10^{6}$ particles; the fitted curve corresponds to $N_{v} / N \sim \exp \left(k w^{-\eta}\right)$, where $k=-15.44$ and $\eta=1.046$. In (b) the alternative model with bifurcation parameter $\beta$ is investigated; the fitted curve corresponds to $N_{v} / N \sim \exp \left(k \beta^{-\eta}\right)$, where $k=-2.807$ and $\eta=0.101$. In these computations, $N=1 \times 10^{6}$ is used for parameters $\beta>2 \times 10^{-6}$, whereas $N=5 \times 10^{6}$ is used for smaller $\beta$. We verified that, even though the particular value of the ratio $N_{v} / N$ depends on where the particles are introduced in the flow, the scaling coefficient $\eta$ is independent of this.

flow model, which has a limiting behavior (for $w \rightarrow 0$ ) closer to that of the realistic system; we find the same results.

In order to investigate the dynamics, we continuously inject particles at a fixed position and plot a superposition of all their trajectories in a stroboscopic manner with the period $T=1$ (i.e., we take snapshots of their positions at integer times). In this way we get a streakline. This is shown in Fig. 1 for $w=2$. We clearly see that the motion near the cylinder's surface on the back of the obstacle is chaotic, with the presence of prominent Kolmogorov-Arnol'd-Moser (KAM) islands. Separation points $S_{+}, S_{-}$and accumulation point $S_{0}$ on the cylinder's surface are also noticeable. 


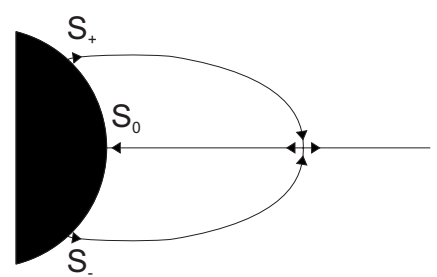

(a)

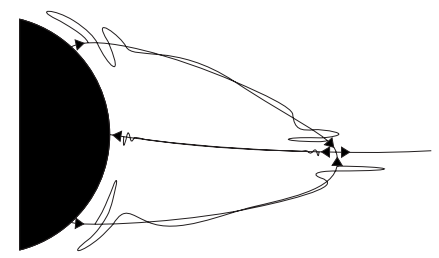

(b)
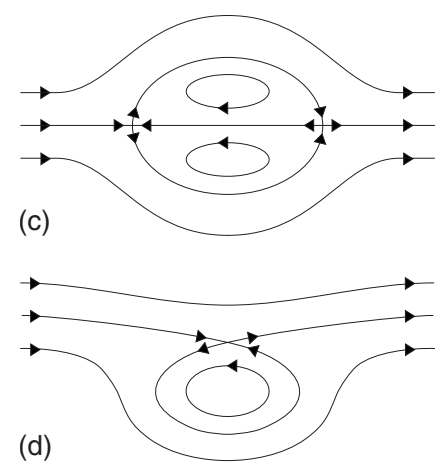

FIG. 3. Schematic picture of the manifolds escaping from the separation points in the flow around an obstacle. In (a), the manifolds are shown for an autonomous system, such that they form separatrices. In (b), the manifolds for a time-periodic flow are depicted, showing a heteroclinic tangle. Images (c) and (d) show the manifolds and streamlines in the case of persistent vortices.

In the region close to the KAM islands the particles whose trajectories get close to the surface of the cylinder exhibit transient chaotic motion. The particles that remain further away from the cylinder do not penetrate this chaotic region and are washed away rapidly by the background flow. To study the probability of particles getting in the chaotic region, a large number $N$ of particles are injected into the flow upstream from the cylinder, and the number $N_{v}$ of those which visit the region near the KAM surfaces is recorded. The ratio $N_{v} / N$ is plotted for different values of $w$ in Fig. 2(a). We observed in our simulations that this ratio smoothly approaches zero as $w$ is decreased to zero. Particles thus penetrate the chaotic region in the wake for any nonzero value of $w$. This means that the transition to chaotic scattering coincides with the transition from stationarity to time dependence: as soon as the flow ceases to be stationary, the advection becomes chaotic, and particles coming from the inflow region can access the chaotic region (albeit only in small numbers for $w$ close to 0 ). For heteroclinic tangles connecting hyperbolic saddle points, one would expect the ratio to scale as $N_{v} / N \sim w^{\gamma}$, where $\gamma=1.5$ for incompressible flows $[10,12]$. However, this relationship does not hold for our simulations. We find that the relation $N_{v} / N \sim \exp \left(k w^{-\eta}\right)$ fits well with our numerical data; see Fig. 2(a). This anoma-

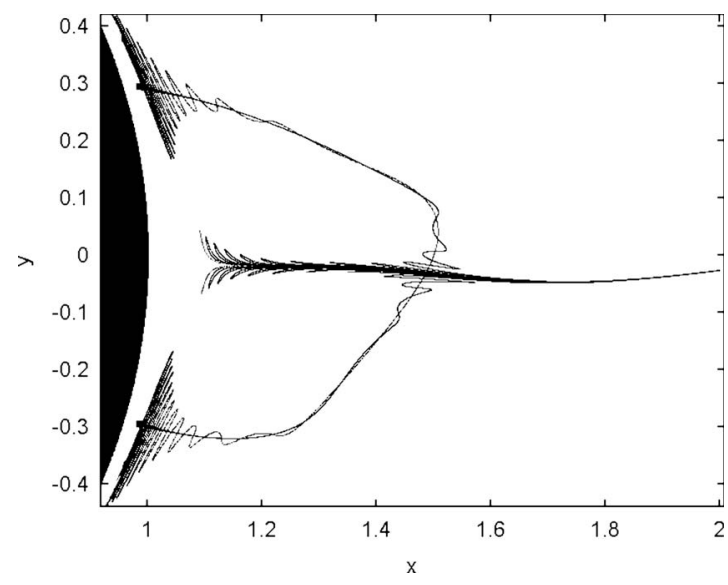

FIG. 4. Visualization of the heteroclinic tangle for $w=2$. Unstable manifolds are visualized by injecting $100 \times 100$ particles close to the separation points and plotting their subsequent positions stroboscopically for 18 periods. The stable manifolds are visualized by injecting $800 \times 800$ particles uniformly in the region $0.9<x$ $<1.6,-0.5<y<0.5$ and checking if they leave downstream (reach $x=+10$ ) or pass through the region $1.25<x<1.35,-0.15<y$ $<0.1$; the boundary between these two outcomes marks the stable manifold of the saddle point.

lous scaling is a direct consequence of the nonhyperbolic nature of the dynamics. We confirmed that the escape time of particles with initial conditions inside the chaotic regions (but outside the KAM islands) scales as a power law, which is characteristic of nonhyperbolic scattering.

To understand the transition between the regular and chaotic advection from a dynamical point of view, we focus on the stagnation points $S_{+}, S_{-}$and accumulation point $S_{0}$ on the downstream surface of the cylinder, depicted in Fig. 3(a). The lateral points $S_{+}$and $S_{-}$have unstable manifolds emanating from them, which act as separatrices at the transition parameter $w=0$, when the flow is stationary. Because of the incompressibility of the flow, there must be a similar point with a stable manifold, and that is the central point $S_{0}$. In this open flow the velocity has to be positive far downstream $(x \rightarrow \infty)$. This implies that the stable manifold of $S_{0}$ cannot extend infinitely far in the downstream direction, otherwise the boundary condition would be violated, as there would be regions of negative flow velocity arbitrarily far downstream. The only way that incompressibility and the downstream boundary condition can be simultaneously satisfied is by the unstable manifolds of $S_{+}$and $S_{-}$joining each other and forming a saddle point, as shown in Fig. 3(a). For the stationary case $w=0$, there are therefore heteroclinic orbits joining the saddle point with the separation points, as shown in Fig. 3(a). These heteroclinic orbits act as separatrices, insulating the inner region near the wall from the outer region. When the flow becomes time dependent for $w>0$, we expect the breakup of the separatrices and the formation of a heteroclinic tangle, which is schematically depicted in Fig. 3(b). From these considerations we thus expect that the scattering becomes chaotic as soon as the flow becomes time dependent; this is indeed what we observe in our simulations. Our reasoning above depends purely on the existence of stagnation points, and our conclusion is a result of the interplay of 

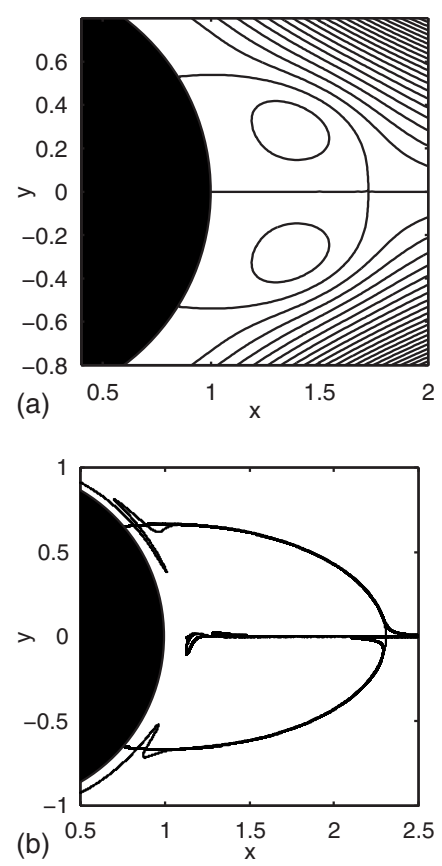

FIG. 5. Results for the modified model. (a) Streamlines of the modified model with $\beta=0$, representing a time-independent flow with two stationary vortices. (b) Heteroclinic tangle for $\beta=0.001$. The stable manifolds of the saddle point and the unstable manifolds of the separation points on the surface of the cylinder were computed in the same way as in Fig. 4.

the incompressible character of the fluid with the openness of the flow with its associated boundary condition. We thus expect the general conclusion to be valid for other flows with stagnant points (or regions). For example, we should have a similar behavior for stagnant or trapped fluid bodies as well, such as the structures depicted in Figs. 3(c) and 3(d).

We calculated the heteroclinic tangle numerically. For the parameter value $w=2$, the manifolds are depicted in Fig. 4. We found that the heteroclinic tangle is present for all $w$ $>0$, consistent with our conclusion described above, that transient chaotic advection appears as soon as the flow becomes time dependent. This is also confirmed in Fig. 2(a), where the ratio of particles accessing the chaotic region behind the cylinder is positive for all $w>0$. As argued above, the fact that the transitions to nonstationarity and to chaotic scattering coincide is a consequence of the presence of stagnant points on the surface of the obstacle. This behavior is qualitatively different from that of flows without stagnant points, which have no separatrices like those shown in Fig. 3.

To verify that our findings are not an artifact of the particular flow model we use, we investigated a different model. In this modified model, there are two stationary vortices present in the time-independent case, as expected in real flows, in contrast to the previous model. This is achieved by replacing Eq. (4) with

$$
\begin{aligned}
g(x, y, t)= & \beta\left[-w h_{1}(t) g_{1}(x, y, t)+w h_{2}(t) g_{2}(x, y, t)\right]+(1-\beta) \\
& \times\left[-w_{s} g_{1 s}(x, y)+w_{s} g_{2 s}(x, y)\right]+u_{0} y s(x, y)
\end{aligned}
$$

where the two new functions are
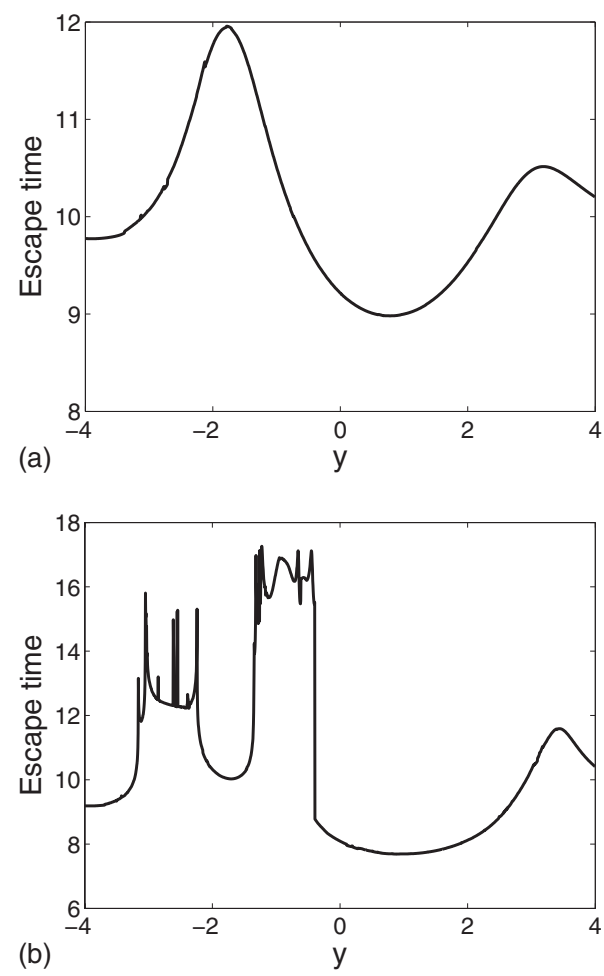

FIG. 6. Escape time for the model of Eq. (8), with initial conditions taken at the $x=0$ segment, as a function of the initial $y$ coordinate. Escape was defined to occur when the particles reach $x=20$, after which they are washed downstream. The parameters are $V=2, \sigma=2, L=5, u=1, k=1$. (a) uses $\mu=2$ and shows regular (nonchaotic) advection, while (b) uses $\mu=10$ and shows chaotic advection.

$$
\begin{aligned}
& g_{1 s}(x, y)=\exp \left\{-R_{0}\left[\left(x-x_{s}\right)^{2}+\alpha_{s}^{2}\left(y-y_{s}\right)^{2}\right]\right\}, \\
& g_{2 s}(x, y)=\exp \left\{-R_{0}\left[\left(x-x_{s}\right)^{2}+\alpha_{s}^{2}\left(y+y_{s}\right)^{2}\right]\right\},
\end{aligned}
$$

describing the contribution to the stream function of two stationary vortices. These vortices are positioned at $x_{s}=1.0$ and $y= \pm y_{s}= \pm 0.3$. The parameters are chosen to be $\alpha_{s}$ $=2.0, w_{s}=6.0$, such that the stationary vortices behave qualitatively as expected in real flows. The value $w=24$ is used in accordance with [2]. The parameter $\beta$ is used in this model as the bifurcation parameter. For $\beta=0$, the flow is stationary, whereas for $\beta=1$ the model is the same as the one used in [2]. The streamlines of this flow for $\beta=0$ are shown in Fig. 5(a).

For all values of $\beta$, we find again a heteroclinic tangle, as depicted in Fig. 5(b) for $\beta=0.001$. This region is accessible for particles injected upstream from the obstacle. By injecting many particles into the flow upstream, the number $N_{v}$ of particles entering the chaotic region is shown in Fig. 2(b) for different values of $\beta$. We see that the ratio of chaotic to all particle trajectories, again, follows $N_{v} / N \sim \exp \left(k \beta^{-\eta}\right)$.

The above analysis is only valid for flows with stagnation points. In flows with no boundaries we may have no stagnation points at the stationary time-dependent transition, and in this situation we expect that advection will be regular immediately after the onset of time dependence. An example of 
boundaryless flows consists of a flow with a uniform velocity field, on which a time-dependent component is superimposed. If the time-dependent part has small amplitude, a particle will be washed away by the constant background flow, and there will be no chaotic advection. Transition to chaos only happens for sufficiently high amplitudes, in contrast to the case with boundaries. An example of such a flow is given by the stream function

$$
\psi=V y+\mu \exp \left(-\frac{(x-L)^{2}+y^{2}}{2 \sigma^{2}}\right) \cos [k(y-u t)],
$$

where $V$ is the constant background velocity, $\mu$ is the strength of the periodic perturbation (so the flow is stationary for $\mu=0), \sigma$ is the characteristic length scale of the perturbation, $k$ is its wave number, and $u$ is its propagation velocity; the period of the perturbation is given by $2 \pi / k u$. This flow is a modification of the one described in Ref. [6], used for modeling ecological flows. Based on the above reasoning, we predict that there is a range of positive $\mu$ for which the flow is time dependent, but the advection is regular, in contrast with the flow with boundaries. A simple way to test this is to plot the escape time of a particle as a function of its initial starting point; this plot should be smooth in the case of regular advection, while it has a Cantor set of singularities where the escape time diverges when advection is chaotic. Figure 6(a) shows that for a small value of $\mu(\mu=2)$, the escape time plot is perfectly regular, and the advection is regular, even though the flow is time dependent; this supports our predictions. As $\mu$ increases, this flow eventually becomes chaotic; this can be seen in Fig. 6(b) (with $\mu=10$ ), which shows the patterns of peaks characteristic of chaotic scattering. Magnifications of regions with peaks in this figure reveal an infinitely fine structure of ever higher peaks, a result of the fact that the peaks lie on a Cantor set. We found that for this system, and for the parameters we used (see caption of Fig. 6), the transition from regular to chaotic advection happens at around $\mu \approx 5$.

In conclusion, we established that fluid flows with stagnant points or regions contain separatrices that are barriers to transport in the stationary case. These separatrices break up as soon as the flow becomes time dependent, resulting in a chaotic sea that can trap for a transient time the particles coming from upstream. Hence there is an immediate transition to chaotic advection in these systems. This behavior is to be contrasted with systems without such stagnant regions: there the lack of separatrices imply that a small timedependent velocity component is not enough to compensate for the background velocity that washes out the particles, and hence time periodicity does not necessarily imply chaos. Due to the presence of the surface and the KAM islands the dynamics around an obstacle is nonhyperbolic, which yields a nontrivial scaling near the bifurcation. As a final remark, we note that, although the stagnant points with zero velocity can only be distinguished for an obstacle with fixed position, a system with a moving obstacle will experience the same dynamics in a comoving coordinate system.

We would like to thank CNPq for partial support of this project. Gy.K. is indebted for financial support from OTKA No. NK 72037.
[1] M. V. Dyke, An Album of Fluid Motion (Parabolic Press, Stanford, 1982).

[2] C. Jung, T. Tél, and E. Ziemniak, Chaos 3, 555 (1993).

[3] A. Péntek, Z. Toroczkai, T. Tél, C. Grebogi, and J. A. Yorke, Phys. Rev. E 51, 4076 (1995).

[4] T. Tél, A. de Moura, C. Grebogi, and G. Károlyi, Phys. Rep. 413, 91 (2005).

[5] A. Wonhas and J. C. Vassilicos, Phys. Rev. E 65, 051111 (2002).

[6] E. Hernández-García and C. López, Ecol. Complexity 1, 253 (2004).

[7] G. Károlyi, A. Péntek, I. Scheuring, T. Tél, and Z. Toroczkai,
Proc. Natl. Acad. Sci. U.S.A. 97, 13661 (2000).

[8] C. López, Z. Neufeld, E. Hernández-García, and P. Haynes, Phys. Chem. Earth, Part B 26, 313 (2001).

[9] M. Cencini, G. Lacorata, A. Vulpiani, and E. Zambianchi, J. Phys. Oceanogr. 29, 2578 (1999).

[10] C. Grebogi, E. Ott, and J. A. Yorke, Phys. Rev. Lett. 57, 1284 (1986).

[11] Y.-C. Lai, C. Grebogi, R. Blümel, and M. Ding, Phys. Rev. A 45, 8284 (1992).

[12] C. Grebogi, E. Ott, F. Romeiras, and J. A. Yorke, Phys. Rev. A 36, 5365 (1987) 